\title{
V024 CONDITIONING A STOCHASTIC MODEL OF FLUVIAL CHANNEL RESERVOIRS USING SEISMIC AND WELL DATA
}

\author{
JOHN INGE BERG' ${ }^{\prime}$, ALISTER MACDONALD', GYRID JOHNSEN ${ }^{2}$ and RAGNAR HAUGE ${ }^{3}$ \\ ' Statoil Research Centre, Postuttak, 7005 Trondheim, Norway \\ ${ }^{2}$ Norsk Hydro Research Centre \\ ${ }^{3}$ Norwegian Computing Centre
}

\section{Background}

Fluvial reservoirs are highly heterogeneous and comprise a complex network of channel-like bodies. To optimise the development of these reservoirs it is important to model the channel distribution between well observations in a realistic manner, and stochastic modelling techniques are becoming increasingly applied for this purpose. This paper presents a stochastic method where seismic data are used to constrain the modelled spatial distribution of channels between wells.

\section{Seismic Response to Fluvial Reservoirs}

The Triassic and Jurassic fluvial channel sand bodies in the North Sea are typically associated with marginally lower acoustic impedance (2-15\% on a seismic scale) when compared with the enclosing overbank mudstones. Individual channel bodies are between $5 \mathrm{~m}$ and $25 \mathrm{~m}$ thick, thicknesses which generally are less than the conventional seismic resolution. The seismic response in the reservoirs is characterised by complex interference patterns. Fluvial channels are also laterally discontinuous and seismic sections are characterised by a lack of continuous reflections and often appear "noisy". Despite these complications, seismic data can often be used to distinguish between volumes of the reservoir which are relatively sand-rich and those which are relatively sand-poor. This is very useful information for constraining the stochastic modelling of the channel distribution.

\section{Concept of Conditional Probabilities}

The information content in seismic data can be quantified using conditional probabilities. Marginal distributions for sand and shale for a seismic attribute are calculated at well locations (Fig. 1). Conditional probabilities are established using the following expression:

$$
\operatorname{Pr}\left(x_{i} \mid s_{i}\right)=\frac{f\left(s_{i} \mid x_{i}\right)}{f\left(s_{i} \mid x_{i=\text { sand }}\right)+f\left(s_{i} \mid x_{i=\text { shale }}\right)}
$$

where $x_{i} \in\{$ sand, shale $\}$ at node $i, s_{i}$ is the seismic attribute value at node $i, f(\cdot \mid \cdot)$ is the experimental marginal distribution density. $\operatorname{Pr}\left(x_{i} \mid s_{i}\right)$ is the conditional probability function when it is defined for the whole data population.

\section{Modelling Algorithm}

The stochastic model is an object model where channel geometry parameters (width, thickness, sinuosity etc.) are described by $1 \mathrm{D}$ Gaussian fields and input as probability functions. The seismic input to the model includes (1) a 3D grid of seismic attributes (e.g. acoustic imperlance) and (2) 
a conditional probability function for channel sandstone given the seismic attribute. A simulated annealing algorithm is then used to generate realisations from the stochastic model by iteratively adding and subtracting channel bodies from the simulation volume. For each iteration the probability of the simulated geometries is calculated according to the geological, seismic, and well-data constraints. The seismic term in the objective function has the form:

$$
\pi(s \mid r) \sim \prod_{i=1}^{N} \pi\left(s_{i} \mid x_{i}\right) \sim \prod_{i=1}^{N} \operatorname{Pr}\left(x_{i} \mid s_{i}\right) \pi\left(s_{i}\right)
$$

where $\pi(s \mid r)$ is the probability for the seismic given the reservoir, and $\pi\left(s_{i}\right)$ is the distribution estimated from the seismic data. Generally an increase in the seismic term will lead to an acceptance of the simulated channel.

\section{Tests}

Synthetic data. The purpose of using synthetic data is twofold: (1) to find a procedure to determine the simulation parameters in a real data case; and (2) to investigate how much the seismic data will affect the apriori distribution of channel geometries.

Real data. Real data were taken from the U.Ness Formation in the Oseberg field in the Norwegian North Sea. Acoustic impedance distributions (Fig. 1) for sand and shales show a clear separation and are thus applicable to lithology prediction. Two cases have been studied; (1) conditioning on well observations and (2) conditioning on seismic impedance and well observations simultaneously.

\section{Conclusions}

For both data sets, synthetic and real, tests show that the realisations honour the seismic data as described by the conditional probability function and the well observations. From multiple realisations the posteriori distributions are updated according to the observations, as will be demonstrated in the synthetic case.

Although seismic data cannot be used for mapping individual sand bodies (in a deterministic sense), the use of seismic data (in a stochastic framework) improves the reservoir model by constraining the distribution of channel bodies between well observations, and by reducing the uncertainty in the reservoir model.
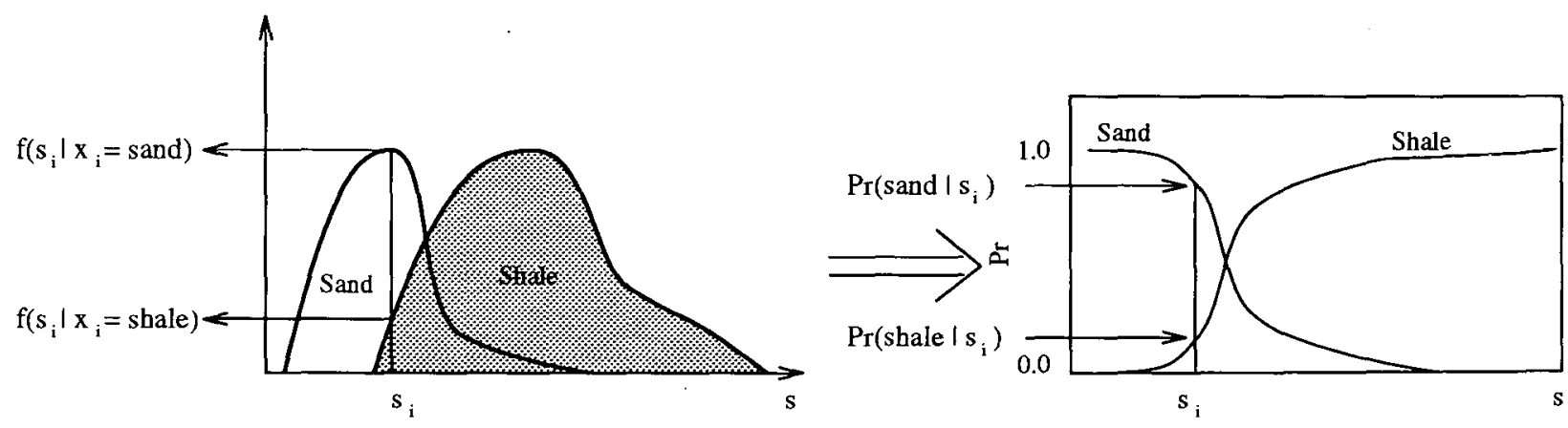

Figure 1: Computing the conditional probability functions (right figure) from marginal distributions (left figure) for sand and shale. See equation 1 for the transformation to probabilities. 